\section{Embarazo e infección por coronavirus (COVID19)}

Pregnancy and coronavirus infection (COVID19)

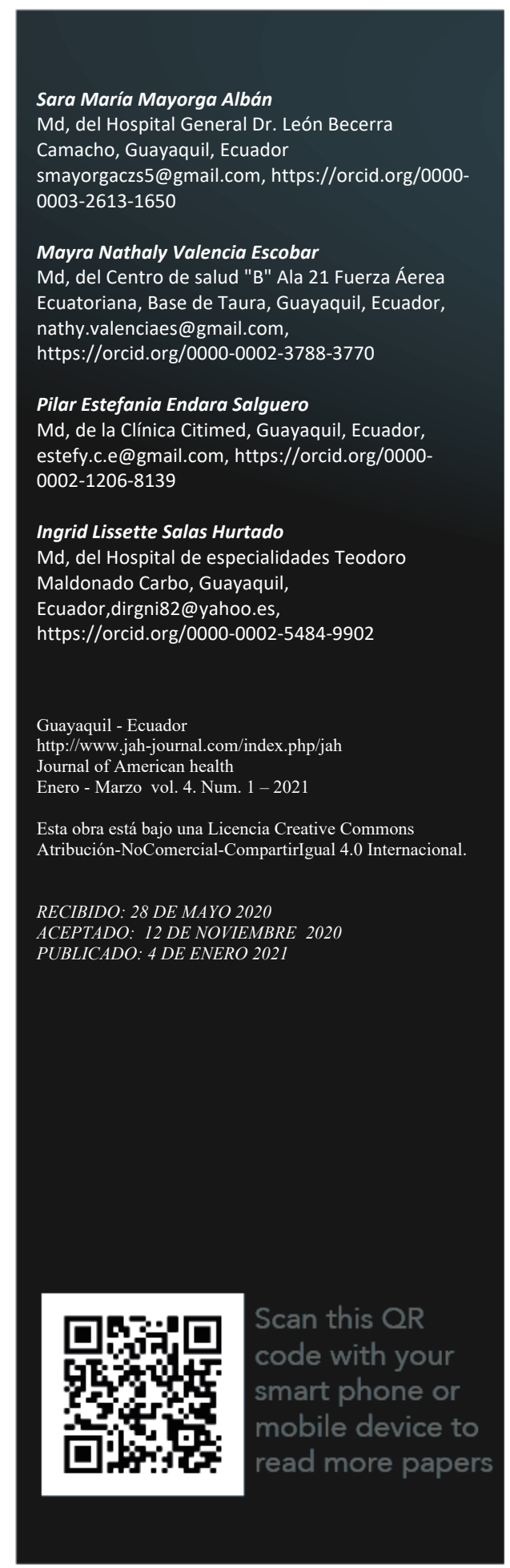

\section{RESUMEN}

La asociación entre el embarazo y COVID-19 sugiere necesidad de aclaraciones, según la OPS se registraron 60 mil 458 casos confirmados de COVID-19 entre mujeres embarazadas, incluyendo 458 muertes $(0,1 \%)$ en 14 países de las Américas; el objetivo de este estudio es actualizar los referentes teóricos sobre la mujer embarazada y su asociación con COVID 19, Se realizó una búsqueda sistemática de investigaciones recientes en bases de datos de Elsevier, Pubmed y Scopus, Google académico de artículos publicados entre el 2019 al 2021, de idioma inglés y español. Un porcentaje mínimo de las gestantes presentarán complicaciones asociadas a la infección por coronavirus SARS CoV-2, sin embargo son más frecuentes en el tercer trimestre, la transmisión vertical es poco frecuente, pero no produce malformaciones fetales, sin embargo se ha descrito mayor riesgo de aborto y retraso del crecimiento intrauterino, por lo tanto, será importante diagnosticarlas y tratarlas de forma precoz; respecto a la terapéutica se usa la heparina para evitar formación de trombos similar a los que ocurre con la población general valorando el riesgo beneficios, la duración del tratamiento variará en función de la gravedad, no obstante se debe garantizar al máximo la seguridad de las pacientes no infectadas e infectadas, para asegurar el bienestar de la madre y el feto. La finalidad de esta revisión es proporcionar las herramientas y claves diagnósticas para la identificación oportuna de la patología, ayude como guía adicional sobre las tendencias por SARS-CoV-2. 
PALABRAS CLAVE: embarazo, complicaciones, terapéutica, COVID 19, aspectos clínicos

\section{ABSTRACT}

The association between pregnancy and COVID19 suggests a great need for clarification. According to PAHO, 60,458 confirmed cases of COVID-19 were registered among pregnant women, including 458 deaths $(0.1 \%)$ in 14 countries of the Americas; The objective of this study is to update the theoretical references on pregnant women and their association with COVID 19, A systematic search of recent research was carried out in Elsevier, Pubmed and Scopus databases of articles published between 2019 and 2021, language English and Spanish. Clinical trials, meta-analyzes, case reports, and data analysis studies were excluded. A minimum percentage of pregnant women will present complications associated with the SARS CoV-2 coronavirus infection, however they are more frequent in the third trimester, vertical transmission appears infrequently, but does not produce fetal malformations, however a greater risk has been shown of abortion and intrauterine growth retardation, for this reason it will be important to diagnose and treat them early; Regarding therapeutics, heparin is used to avoid thrombus formation similar to those that occurs in the general population, assessing the risk benefits, the duration of treatment will vary depending on the severity despite not being susceptible to this morbidity, however it should ensure the maximum safety of uninfected and infected patients, to ensure the well-being of both. The purpose of this review is to provide the tools and diagnostic keys for the timely identification of the pathology, to help as an additional guide on the trends for SARS-CoV-2 and the severity of the disease among pregnant women.

KEYWORDS: pregnancy, complications, therapy, COVID 19, clinical aspects

\section{INTRODUCCIÓN}

La Infección por SARS-CoV-2. afecta principalmente a personas de 30 a 79 años, siendo muy poco frecuente en menores de 20 años y las complicaciones están determinadas por ciertos factores de riesgos como edad, enfermedades concomitantes, cepas, carga viral entre otras.

Las mujeres embarazadas infectadas por COVID-19, como el resto de personas pueden padecer estas comorbilidades y presentar síntomas relacionados con la población general como fiebre, tos y dificultad para respirar. Los problemas respiratorios aparecen cuando la infección afecta a los pulmones y causa una neumonía atípica.

En el 2009, las mujeres embarazadas representaron el $1 \%$ de los pacientes infectados con el virus de la influenza A subtipo $\mathrm{H} 1 \mathrm{~N} 1$, y el $5 \%$ de muertes relacionadas a dicho agente viral. También está documentado que el SARS-CoV y el MERS-CoV son responsables de complicaciones graves durante el embarazo, con necesidad de intubación endotraqueal, 
insuficiencia renal y muerte, no obstante, no demostraron una relación causal clara con estas complicaciones. (1)

Las embarazas no parecen tener una mayor susceptibilidad para infectarse por coronavirus, ni para presentar complicaciones graves. Un pequeño porcentaje presentarán una complicación asociada a la infección por coronavirus SARS CoV-2, por este motivo será importante diagnosticarlas y tratarlas de forma precoz (2).

De acuerdo con lo que se conoce hasta el momento, las mujeres embarazadas tienen mayor riesgo de enfermarse gravemente y morir a causa del COVID-19 que las personas que no están embarazadas. Además, las personas embarazadas con COVID-19 podrían tener mayor riesgo de presentar otros resultados adversos, como el nacimiento prematuro (3).

Pero, datos actualizados sobre covid-19 y embarazo sugieren que no existe mayor riesgo de aborto o pérdida gestacional precoz en gestantes con COVID-19. Al no existir evidencia de transmisión intrauterina es muy poco probable que la infección por COVID-19 pueda producir defectos congénitos (1). Sin embargo la elevada contagiosidad por este virus es recomendable que el personal de salud que atiende a embarazadas siga de forma estricta las medidas de prevención recomendadas en los protocolos específicos (bata, guantes, gorro, gafas y mascarilla quirúrgica o N95) según tipo de exposición y posterior a la atención se proceda a la limpieza del material utilizado (cardiotocógrafos, ecógrafos y resto de material) siguiendo la norma establecida afín de evitar infectar o infectarse mediante esta exposición (1).

En virtud de este antecedente se pretende proporcionar una revisión actualizada de los conceptos más importantes sobre la mujer embarazada y la infección por COVID-19 y establecer los efectos sobre este grupo vulnerable y sobre el feto.

\section{MATERIALES Y MÉTODOS}

Se realizó una búsqueda bibliográfica en Google académico, PubMed y Scielo, Scopus de los últimos años previos a esta publicación sobre los aspectos clínicos de la mujer embarazada con infección por covid-19. Se incluyeron estudios de revisión narrativa o sistemática utilizando las palabras claves "embarazo", "manifestaciones clínicas" "COVID 19", "patogenia", "Complicaciones" "terapéutica", publicaciones escritas en idioma inglés o español entre el 2019 y 2021. Se excluyeron los artículos sobre cartas a los editor y memorias de congresos. Se proyectó un total de 40 artículos, de los cuales se seleccionaron 30 investigaciones completas. De estos, 11 artículos no eran elegibles, ya que no contenían evaluación clínica, diagnóstica, complicaciones y terapéutica y, por lo tanto, se excluyeron.

\section{RESULTADOS}

La tasa de letalidad de la infección por SARS-CoV entre mujeres embarazadas es del $25 \%$, sin embargo, no hay evidencia clara de que las mujeres embarazadas sean más susceptibles a la infección por COVID-19 o que aquellas con infección sean más propensas a desarrollar neumonía grave (1). 
Por la condición de hipercoagulabilidad propia del embarazo, toda gestante COVID - 19 positiva cuyo cuadro clínico sea moderado, es decir, "compromiso de vías respiratorias inferiores con disnea, imágenes radiológicas sugestivas de neumonía en < 50\% del área pulmonar, fiebre >a 38.5 grados que no responde a paracetamol y saturación de oxígeno mayor del 93\%", deberá ser hospitalizada y recibir terapia profiláctica preferiblemente con heparina de bajo peso molecular (HBPM) (1).

\section{Diagnostico}

Respecto al diagnóstico métodos, clasificación clínica, se sigue las normas generales, por ejemplo, los métodos bioquímicos y test serológicos. Sin embargo, precisa considerar ciertos aspectos en la mujer embarazada.

El retraso del crecimiento intrauterino podría ser una posible complicación a largo plazo en pacientes que se recuperan de la infección por COVID-19.

Los estudios de imagen como la Radiografía simple de tórax en diferentes proyecciones resultan esencial para la evaluación de la situación clínica de una embarazada con COVID-19. En caso de indicación diagnóstica también se podrá realizar una tomografía computarizada torácica. Para ambas exploraciones se debe obtener el consentimiento informado (toma de decisiones compartidas) y se utilizarán las medidas habituales de protección fetal (delantal abdominal de plomo) (1).

Las mujeres embarazadas con infección confirmada o en recuperación de la enfermedad debe tener una exploración de crecimiento cada 4 semanas después de la recuperación. Las exploraciones de seguimiento deben programarse de acuerdo con los hallazgos.

Asegurarse sobre las medidas preventivas como higiene de manos, desinfección de superficies y adherirse a las medidas de distanciamiento social.

\section{Infección congénita:}

Se han realizado estudios para determinar presencia de trasmisión vertical. De hecho, un estudio retrospectivo realizado en el Hospital Zhongnan de la Universidad de Wuhan analizó nueve casos de pacientes embarazadas con neumonía por COVID-19, en tercer trimestre de embarazo y todas fueron sometidas a parto por cesárea por indicación obstétrica, ninguna con comorbilidades asociadas (4).

En muestras de líquido amniótico, sangre de cordón umbilical, leche materna y de hisopo orofaríngeo de los recién nacidos fueron negativas en 6 casos en un estudio de gestantes positiva a SARS-CoV-2 (5). Otros estudios previos no han reportado evidencia de infección congénita y actualmente no hay datos sobre el riesgo de malformación congénita cuando la infección por COVID-19 se adquiere durante el primer trimestre del embarazo. Sin embargo, una exploración 
detallada de la morfología está indicada para mujeres embarazadas que tuvieron infección confirmada por COVID-19 (1).

\section{Aspectos clínicos y Gravedad:}

Los síntomas de pacientes embarazadas y que cursan con infección por SARS-CoV2 son similares y diversos al igual que en pacientes no gestantes, los síntomas más frecuentes son fiebre y tos (6).

Las embarazadas infectadas con COVID-19 que no estén de parto y que estén asintomáticas o con síntomas leves, pueden realizar aislamiento en el domicilio durante 10-14 días. Los controles obstétricos, de acuerdo a cada caso, se aplazarán hasta que se finalice el aislamiento. En caso de no poder prorrogar el control obstétrico se realizará en consultas externas (2).

La labor de parto prematura reportados en los estudios no se relacionó con la infección por SARS-CoV2 sino con enfermedades ginecológicas como preeclampsia, mortinatos previos y ruptura prematura de membranas. (6) De hecho, en un metaanálisis de 77 estudios publicado BJM resultó un mayor riesgo de parto prematuro y una mayor necesidad de requerir ventilación invasiva en comparación con las mujeres no embarazadas con SARS-CoV-2, especialmente en aquellas con un trastorno médico subyacente (7).

En España los estudios en la embarazada y del recién nacido en relación al COVID-19 señala como síntoma principal a la fiebre, que no siempre aparece antes del nacimiento del neonato (5). Con la evidencia actual disponible, las mujeres embarazadas parecen tener un curso similar de la enfermedad, a la población general. Sin embargo, brotes anteriores con virus respiratorios similares (SARS, MERS) han demostrado que esta población, era más vulnerable en el segundo y tercer trimestre. Por lo tanto, hasta que se disponga de más datos, las embarazadas con edad gestacional mayor de 24 semanas, deben estar estrictamente protegidas contra la infección (8).

La literatura más reciente señala que la infección por COVID-19 se caracteriza por generar un aumento importante de citoquinas a nivel sistémico como la IL-2, IL-7, IL-10, factor de necrosis tumoral alfa, factor estimulante de colonias granulocíticas, por lo que las mujeres embarazadas que se encuentran bajo un estado pro-inflamatorio podrían presentar una respuesta más severa ante dicha infección (8), (4).

Durante el tercer trimestre de embarazo los depósitos de fibrina alrededor de las vellosidades son un hallazgo esperado que no traduce relevancia clínica debido a la restante reserva placentaria funcional; sin embargo, en estas pacientes además de dichas observaciones se documentó un importante aumento de depósitos de fibrina a nivel del estroma velloso. Lo anterior sugiere que la infección por COVID-19 podría causar disminución del flujo sanguíneo a nivel placentario y por ende generar hipoxia que llevaría a microinfartos de este tejido (7), (4). 
Todas las pacientes embarazadas deben ser evaluadas por la presencia de fiebre y sintomatología respiratoria, idealmente previo a la llegada de la paciente a un centro de atención de salud. En caso de presentar sintomatología respiratoria deberían contactar vía telefónica al centro o profesional de salud encargado para evaluar la severidad de los síntomas y garantizar que sean separadas de otras pacientes en salas de espera y se les brinde una mascarilla cuando ingresen al centro de salud (4), (4).

\section{Terapéutica:}

La embarazadas asintomáticas o que presentan síntomas leves, deben el aislarse en domicilio durante 10-14 días ya que no es indicación de hospitalización, si han tenido contacto epidemiológico lo ideal es que realicen pruebas diagnósticas por medio de PCR.

Las mujeres embarazadas con enfermedad severa, deben recibir oxigenoterapia cuando están con dificultad respiratoria, hipoxemia o shock. Se debe iniciar con oxígeno por nasocánula a 5 L/minuto e ir titulando el flujo. Para las pacientes en condición crítica se puede utilizar la mascarilla con reservorio a 10-15 L/min durante RCP. La meta de saturación de oxígeno para la paciente embarazada estable ronda entre 92 a 95\%. Una vez que la madre esté estable, se debe evaluar el bienestar fetal. El monitoreo de estas pacientes buscando signos de deterioro clínico (sepsis, falla respiratoria rápidamente progresiva) debe ser periódico y estricto (4).

La sola infección por SARS-CoV-2 no es una indicación de interrupción del embarazo, sobre todo si la madre no presenta criterios de gravedad (13). La indicación de interrumpir el embarazo depende del estado de la enfermedad, la edad gestacional y viabilidad fetal; además es vital un abordaje multidisciplinario para la toma de decisiones (14), (4). El momento y la vía del parto se individualizará según el estado de la madre, las semanas de embarazo y las condiciones obstétricas (2).

De no tener una indicación clara para interrupción del embarazo, se ha propuesto que las mujeres embarazadas, por los cambios fisiológicos propios del embarazo, pueden sufrir un deterioro clínico más rápido y, al desconocer el efecto del virus y los antivirales en el desarrollo fetal, en embarazos a término se recomienda el parto lo más pronto posible (1). Aún no está claro cuál es la vía de parto más segura para una paciente con infección por SARS-COV-2 (4).

\section{Discusión:}

Según los resultados de búsquedas bibliográfica, las mujeres embarazadas con COVID19 parecen tener más probabilidad de desarrollar complicaciones respiratorias, con ingreso a cuidado intensivo que las mujeres que no gestantes, según los CDC, además la raza negra o hispanas parecen estar afectadas de manera desproporcionada con la infección (9), pero los datos existentes son limitados. Cada una de las embarazadas deben considerarse de alto riesgo por la mayor susceptibilidad debido a una respuesta inmune alterada, especialmente en tercer trimestre. Se ha observado aumento de la prevalencia de cesarías respecto a la población gestante general, y por causas todavía no conocida, se ha propuesto que las mujeres 
embarazadas, pueden sufrir un deterioro clínico más rápido y, al desconocer el efecto del virus y los antivirales en el desarrollo fetal, en embarazos a término se recomienda el parto lo más pronto posible. Los estudios científicos disponibles al momento han reportado algunos casos de posible transmisión intrauterina o perinatal, pero ésta es muy poco frecuente y no parece que la infección por COVID-19 pueda producir malformaciones fetales Los pocos casos conocidos en neonatos que han adquirido la infección los primeros días son leves. No constan registros de casos graves (2).

Respecto a las complicaciones del primer trimestre y los datos sobre teratología aún no están documentadas, sin embargo, en base a que las células en la interfaz fetal-materna son menos susceptibles a la infección por COVID-19, se estima que el riesgo de complicaciones en el primer trimestre es bajo. Por lo tanto, se recomienda que a las embarazadas que se les informe sobre los riesgos bajos o inexistentes de infección intrauterina por el virus COVID-19 (8). No obstante, según el CDC 2020, las mujeres embarazadas con COVID-19 podrían tener mayor riesgo de registrar resultados adversos durante el embarazo, como el nacimiento prematuro. (10).

En una reciente revisión sistemática que incluyen a 79 mujeres de las cuales 41 (51,9\%) estaban infectadas por COVID-19, 12 (15,2\%) por MERS y 26 (32,9\%) por SARS. Los síntomas más comunes fueron fiebre $(82,6 \%)$, tos $(57,1 \%)$ y disnea el $27,0 \%$. Las incidencias obstétricas en todas las infecciones por CoV 2, fueron: aborto espontáneo en el $32,1 \%$, parto prematuro $(<37$ semana) $24,3 \%$, rotura de membranas $20,7 \%$, PE $16,2 \%$, y retraso de crecimiento fetal $11,7 \%$. El $84 \%$ fueron partos por cesárea y la tasa de muerte perinatal fue el $11,1 \%$. En las gestantes afectas de COVID-19, la complicación obstétrica más frecuente fue el parto prematuro $(<37$ semanas) en el $41 \%$ de los casos y la tasa de muerte perinatal fue el $7 \%$, y en ninguno de los 41 recién nacidos se demostró la transmisión vertical del virus. (8)

Respecto a fármacos aún no está del todo claro sus beneficios en relación a los corticosteroides solo tienen un lugar en la prevención de la hipoplasia pulmonar neonatal, la enterocolitis necrótica y la hemorragia interventricular debido a la prematuridad. Los antipalúdicos (hidroxicloroquina) tiene un perfil de seguridad razonable en el embarazo, pero se deben tomar precauciones generales, sin embargo, es conocido las limitaciones de los estudios con estos fármacos usados en esta virosis, y los antirretrovirales no tienen indicación (11).

\section{CONCLUSIONES}

La embarazado no es una persona inmunodeprimida, sin embargo, es conocido que las adaptaciones fisiológicas de la madre al embarazo condicionan a ciertas susceptibilidades de patógenos; en este contexto el COVID 19 debe considerar complicaciones como el retraso de crecimiento intrauterino, abortos, prematuridad, pero no reporta malformaciones congénitas, respecto al diagnóstico y tratamiento debe considerarse aspectos relacionados a la población general y como aún no se ha establecido con seguridad un tratamiento para COVID-19, todos los ensayos farmacológicos deben considerarse experimentales, y esto debe explicarse a este grupo, por esto es transcendental identificar los factores de riesgo de enfermedad grave a causa del COVID-19, describir el uso de terapéutica de investigación y no aprobada, y evaluar 
los efectos del COVID-19 sobre los resultados en el embarazo y los neonatos asi como su riesgo beneficio.

\section{REFERENCIAS}

1. Páez LS, Novoa S, Nieto B, Yépez E. LINEAMIENTOS DE MANEJO ANTE LA INFECCIÓN POR COVID19 DURANTE EL EMBARRAZO Quito: SOCIEDAD ECUATORIANA DE GINECOLOGÍA Y OBSTETRICIA; 2020.

2. Sant Joan de Déu Barcelona Hostipal. Sant Joan de Déu Barcelona Hostipal. [Online].; 2020. Available from: https://www.sjdhospitalbarcelona.org/es/coronavirus-embarazo-informacionfiable-para-pacientes-durante-gestacion-parto-posparto.

3. CDC. Centros para el Contol y la Prevención de Enfermedades. [Online].; 2020. Available from: https://espanol.cdc.gov/coronavirus/2019-ncov/need-extra-precautions/pregnancybreastfeeding.html.

4. Herrera Pérez JC, Montero Fonseca J, Campos Sánchez S. COVID-19 y embarazo: revisión de la bibliografía actual. Revista Médica Sinergia. Vol. 5 Num. 9. 20200.

5. Caparros-Gonzalez RA. Consecuencias maternas y neonatales de la infección por coronavirus Covid-19 durante el embarazo: una scoping review. Rev. Esp. Salud Publica 94. 2020.

6. Armijos G, Salazar J. COVID- 19 y embarazo. Guayaquil:; 2020.

7. Infomed. Infomed. [Online].; 2020 [cited 2021 Enero. Available from: https://temas.sld.cu/coronavirus/2020/10/20/embarazo-y-sars-cov-2/.

8. González AO. Embarazo en paciente COVID-19. Ourense:; 2020.

9. Universitat de Barcelona. PROTOCOLO: CORONAVIRUS (COVID-19) Y GESTACIÓN Barcelona: Universitat de Barcelona; 2020.

10. Centro de prevencion enefremdades. INVESTIGACION DE IMPACTO COVOD 19 EMBARAZADAS. [Online].; 2020 [cited 2021 enero. Available from: https://espanol.cdc.gov/coronavirus/2019ncov/cases-updates/special-populations/pregnancy-data-on-covid-19/what-cdc-is-doing.html.

11. González AO. EMBARAZO Y COVID-19. Hospital Universitario de Galicia. 2020 May. 